\title{
Front Matter: Volume 8380
}

, "Front Matter: Volume 8380," Proc. SPIE 8380, Atmospheric Propagation IX, 838001 (7 June 2012); doi: 10.1117/12.979459

SPIE Event: SPIE Defense, Security, and Sensing, 2012, Baltimore, Maryland, United States 


\section{PROCEEDINGS OF SPIE}

\section{Atmospheric Propagation IX}

Linda M. Wasiczko Thomas

Earl J. Spillar

Editors

25-26 April 2012

Baltimore, Maryland, United States

Sponsored and Published by

SPIE

Volume 8380

Proceedings of SPIE, 0277-786X, v. 8380

SPIE is an international society advancing an interdisciplinary approach to the science and application of light.

Atmospheric Propagation IX, edited by Linda M. Wasiczko Thomas, Earl J. Spillar, Proc. of SPIE Vol. 8380 $838001 \cdot$ ㅇ 2012 SPIE · CCC code: 0277-786X/12/\$18 - doi: 10.1117/12.979459 
The papers included in this volume were part of the technical conference cited on the cover and title page. Papers were selected and subject to review by the editors and conference program committee. Some conference presentations may not be available for publication. The papers published in these proceedings reflect the work and thoughts of the authors and are published herein as submitted. The publisher is not responsible for the validity of the information or for any outcomes resulting from reliance thereon.

Please use the following format to cite material from this book:

Author(s), "Title of Paper," in Atmospheric Propagation IX, edited by Linda M. Wasiczko Thomas, Earl J. Spillar, Proceedings of SPIE Vol. 8380 (SPIE, Bellingham, WA, 2012) Article CID Number.

ISSN 0277-786X

ISBN 9780819490582

Published by

SPIE

P.O. Box 10, Bellingham, Washington 98227-0010 USA

Telephone +1 3606763290 (Pacific Time) · Fax +1 3606471445

SPIE.org

Copyright () 2012, Society of Photo-Optical Instrumentation Engineers

Copying of material in this book for internal or personal use, or for the internal or personal use of specific clients, beyond the fair use provisions granted by the U.S. Copyright Law is authorized by SPIE subject to payment of copying fees. The Transactional Reporting Service base fee for this volume is $\$ 18.00$ per article (or portion thereof), which should be paid directly to the Copyright Clearance Center (CCC), 222 Rosewood Drive, Danvers, MA 01923. Payment may also be made electronically through CCC Online at copyright.com. Other copying for republication, resale, advertising or promotion, or any form of systematic or multiple reproduction of any material in this book is prohibited except with permission in writing from the publisher. The CCC fee code is 0277-786X/12/\$18.00.

Printed in the United States of America.

Publication of record for individual papers is online in the SPIE Digital Library.

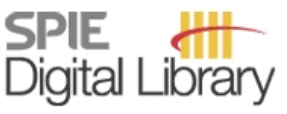

SPIEDigitalLibrary.org

Paper Numbering: Proceedings of SPIE follow an e-First publication model, with papers published first online and then in print and on CD-ROM. Papers are published as they are submitted and meet publication criteria. A unique, consistent, permanent citation identifier (CID) number is assigned to each article at the time of the first publication. Utilization of CIDs allows articles to be fully citable as soon as they are published online, and connects the same identifier to all online, print, and electronic versions of the publication. SPIE uses a six-digit CID article numbering system in which:

- The first four digits correspond to the SPIE volume number.

- The last two digits indicate publication order within the volume using a Base 36 numbering system employing both numerals and letters. These two-number sets start with 00, 01, 02, 03, 04 , $05,06,07,08,09,0 A, 0 B \ldots$. OZ, followed by 10-1Z, 20-2Z, etc.

The CID number appears on each page of the manuscript. The complete citation is used on the first page, and an abbreviated version on subsequent pages. Numbers in the index correspond to the last two digits of the six-digit CID number. 


\section{Contents}

vii Conference Committee

\section{SESSION 1 LASER COMMUNICATIONS I}

838002 Retro-reflector diversity effects in free-space optical links [8380-01]

W. S. Rabinovich, R. Mahon, M. F. Callahan, C. Moore, M. Suite, M. Ferraro, P. G. Goetz, U.S. Naval Research Lab. (United States)

838003 Miniature lasercomm module for integration into a small unmanned aerial plafform [8380-02]

M. J. Vilcheck, H. R. Burris, C. I. Moore, W. R. Smith IV, L. M. Thomas, U.S. Naval Research Lab. (United States)

838004 Fade and surge asymmetry of direct single-mode-fiber coupled free-space optical signal under weak atmospheric turbulences [8380-03]

Y. Arimoto, National Institute of Information and Communications Technology (Japan)

838005 Automating a lasercomm terminal on a small unmanned aerial platform [8380-04] W. R. Smith IV, H. R. Burris, M. J. Vilcheck, C. I. Moore, L. L. Summers, L. M. Thomas, U.S. Naval Research Lab. (United States)

838006 Design simulation and analysis of a fiber-bundle based optical wireless link [8380-05]

P. G. LoPresti, Univ. of Tulsa (United States); D. Zhou, Univ. of Oklahoma (United States); Z. Shi, Univ. of Tulsa (United States); H. H. Refai, Univ. of Oklahoma (United States)

\section{SESSION 2 LASER COMMUNICATIONS II}

838007 Analysis of link performance for the FOENEX laser communications system (Invited Paper) [8380-06]

J. C. Juarez, D. W. Young, R. A. Venkat, D. M. Brown, A. M. Brown, The Johns Hopkins Univ. Applied Physics Lab (United States); R. L. Oberc, General Dynamics (United States); J. E. Sluz, The Johns Hopkins Univ. Applied Physics Lab. (United States); H. A. Pike, Defense Strategies and Systems, Inc. (United States); L. B. Stotts, Stotts Consulting, LLC (United States)

838008 Development of a large area InGaAs APD receiver based on an impact ionization engineered detector for free-space lasercomm applications [8380-07]

H. R. Burris, M. S. Ferraro, U.S. Naval Research Lab. (United States); W. T. Freeman, Smart Logic Inc. (United States); C. I. Moore, J. L. Murphy, W. S. Rabinovich, W. R. Smith, L. L. Summers, L. M. Thomas, M. J. Vilcheck, U.S. Naval Research Lab. (United States); W. R. Clark, W. D. Waters, OptoGration, Inc. (United States)

838009 High-performance free-space optical modem hardware [8380-08]

J. E. Sluz, J. C. Juarez, C.-H. Bair, R. L. Oberc, R. A. Venkat, D. Rollend, D. W. Young, The Johns Hopkins Univ. Applied Physics Lab. (United States) 
8380 OA Characterization of InGaAs avalanche photodiode arrays with varying geometries for free-space optical communication [8380-09]

M. S. Ferraro, H. R. Burris, R. Mahon, W. S. Rabinovich, U.S. Naval Research Lab. (United States); W. T. Freeman, Smart Logic, Inc. (United States); J. L. Murphy, P. G. Goetz, C. I. Moore, L. M. Thomas, U.S. Naval Research Lab. (United States); W. R. Clark, W. D. Waters, K. Vaccaro, B. D. Krejca, Optogration, Inc. (United States)

8380 OB Evaluation of optical transceivers for mobile FSO applications [8380-10]

D. Zhou, Univ. of Oklahoma (United States); Z. Shi, W. Yi, P. LoPresti, Univ. of Tulsa (United States); H. Refai, Univ. of Oklahoma (United States)

SESSION $3 \quad$ LASER COMMUNICATIONS III

8380 OC Improved atmospheric characterization for free-space link analysis using numerical weather prediction [8380-11]

B. D. Felton, P. D. Hayes, R. J. Alliss, Northrop Grumman Corp. (United States)

\section{SESSION $4 \quad$ ADAPTIVE OPTICS SYSTEMS}

8380 OD Holographic adaptive laser optics system (HALOS) [8380-12]

G. Andersen, F. Ghebremichael, J. Baker, R. Gaddipati, P. Gaddipati, HUA Inc. (United States)

8380 OE Validity of using Gaussian Schell model for extended beacon studies [8380-13] S. Basu, S. J. Cusumano, M. W. Hyde, M. A. Marciniak, S. T. Fiorino, Air Force Institute of Technology (United States)

8380 OF High contrast imaging in the presence of turbulence [8380-14] B. A. Sickmiller, D. W. Oesch, SAIC (United States); D. J. Sanchez, P. R. Kelly, Air Force Research Lab. (United States)

\section{SESSION 5 TURBULENCE}

8380 OG Near-surface turbulent temperature variances and anisotropy at multiple scales of motion [8380-15]

C. Klipp, U.S. Army Research Lab. (United States)

8380 ol Automation of $C_{n}^{2}$ profile extraction from weather radar images [8380-17] L. R. Burchett, S. T. Fiorino, M. Buchanan, Air Force Institute of Technology (United States)

8380 0J Turbulence characterization and image processing data sets from a NATO RTO SET 165 trial in Dayton, Ohio, USA [8380-18]

M.-T. Velluet, ONERA (France); M. Vorontsov, Univ. of Dayton (United States); P. Schwering, TNO (Netherlands); G. Marchi, Fraunhofer-Institut für Optronik, Systemtechnik und Bildauswertung (Germany); S. Nicolas, Norwegian Defence Research Establishment (Norway); J. Riker, U.S. Air Force Research Lab. (United States) 
8380 OM Propagation of laser light through aero-optic flow: dry air at 0.4 Mach with three-dimensional turret [8380-21]

R. L. Beauchamp, S. T. Fiorino, Air Force Institute of Technology (United States)

\section{SESSION 6 ATMOSPHERIC PROPAGATION THEORY}

8380 ON The phase Strehl cumulative distribution function (CDF) in the performance modeling of adaptive optics systems [8380-22]

T. C. Farrell, Air Force Research Lab. (United States)

838000 The aggregate behavior of branch points: verification in wave optical simulation I [8380-24]

D. W. Oesch, SAIC (United States); C. M. Tewksbury-Christle, D. J. Sanchez, P. R. Kelly, Air Force Research Lab. (United States)

8380 OP The aggregate behavior of branch points: theoretical calculation of branch point velocity [8380-25]

D. J. Sanchez, Air Force Research Lab. (United States); D. W. Oesch, SAIC (United States);

P. R. Kelly, Air Force Research Lab. (United States)

8380 OR Using Maple and special functions to study the propagation of coherent light beams inside the Earth-ionosphere waveguide with turbulent media [8380-27]

S. Montoya Isaza, J. F. Ospina Giraldo, Univ. EAFIT (Colombia)

\section{SESSION 7 CLOUDS, AEROSOLS, AND BULK EFFECTS}

8380 OS The mitigation of cloud impacts on free-space optical communications (Invited Paper) [8380-28]

R. J. Alliss, B. Felton, Northrop Grumman Corp. (United States)

8380 ОT Potential impacts of elevated aerosol layers on high energy laser aerial defense engagements [8380-29]

S. T. Fiorino, S. M. Shirey, M. F. Via, D. J. Grahn, M. J. Krizo, Air Force Institute of Technology (United States)

$8380 \mathrm{OU}$ Assimilation of nontraditional datasets to improve atmospheric compensation [8380-30] M. A. Kelly, K. Osei-Wusu, T. S. Spisz, S. Strong, The Johns Hopkins Univ. Applied Physics Lab. (United States); N. Setters, U.S. Air Force (United States); D. M. Gibson, The Johns Hopkins Univ. Applied Physics Lab. (United States)

8380 OV Atmospheric propagation properties of various laser systems [8380-31] G. A. Pitz, Air Force Research Lab. (United States); S. Glass, Haverford College (United States); B. Kamer, Air Force Research Lab. (United States); W. L. Klennert, The Boeing Co. (United States); D. A. Hostutler, Air Force Research Lab. (United States) 
POSTER SESSION

8380 OY Mobile free-space optical communications: a feasibility study of various battlefield scenarios [8380-34]

A. Harris, Univ. of North Florida (United States); M. K. Al-Akkoumi, J. J. Sluss, Jr., The Univ. of Oklahoma (United States)

Author Index 


\title{
Conference Committee
}

\author{
Symposium Chair
}

Kevin P. Meiners, Office of the Secretary of Defense (United States)

Symposium Cochair

Kenneth R. Israel, Lockheed Martin Corporation (United States)

Conference Chairs

Linda M. Wasiczko Thomas, U.S. Naval Research Laboratory (United States)

Earl J. Spillar, Air Force Research Laboratory (United States)

Program Committee

Ammar Al-Habash, Raytheon Space \& Airborne Systems (United States)

Gary J. Baker, Lockheed Martin Space Systems Company (United States)

Harris R. Burris, Jr., U.S. Naval Research Laboratory (United States)

Gary G. Gimmestad, Georgia Tech Research Institute (United States)

Ken Grant, Defence Science and Technology Organisation (Australia)

Juan C. Juarez, The Johns Hopkins University Applied Physics Laboratory (United States)

Christopher I. Moore, U.S. Naval Research Laboratory (United States)

Jonathan M. Saint Clair, The Boeing Company (United States)

David H. Tofsted, U.S. Army Research Laboratory (United States)

Morio Toyoshima, National Institute of Information and

Communications Technology (Japan)

Cynthia Y. Young, University of Central Florida (United States)

Session Chairs

1 Laser Communications I

Juan C. Juarez, The Johns Hopkins University Applied Physics Laboratory (United States)

2 Laser Communications II

Linda M. Wasiczko Thomas, U.S. Naval Research Laboratory (United States) 
3 Laser Communications III

Linda M. Wasiczko Thomas, U.S. Naval Research Laboratory (United States)

$4 \quad$ Adaptive Optics Systems

Earl J. Spillar, Air Force Research Laboratory (United States)

5 Turbulence

Earl J. Spillar, Air Force Research Laboratory (United States)

$6 \quad$ Atmospheric Propagation Theory

Gary J. Baker, Lockheed Martin Space Systems Company (United States)

7 Clouds, Aerosols, and Bulk Effects

David H. Tofsted, U.S. Army Research Laboratory (United States) 